Article

\title{
Longitudinal Monitoring of Alpha-Fetoprotein by Dried Blood Spot for Hepatoblastoma Screening in Beckwith-Wiedemann Syndrome
}

\author{
Alessandro Mussa ${ }^{1,2, * \mathbb{D}}$, Valentina Pia Ciuffreda ${ }^{1}$, Pina Sauro ${ }^{1}$, Veronica Pagliardini ${ }^{1}$, \\ Severo Pagliardini ${ }^{1}$, Diana Carli ${ }^{1}{ }^{\circledR}$, Jennifer M. Kalish ${ }^{3}$, Franca Fagioli ${ }^{1,4}$, Enza Pavanello ${ }^{1}$ \\ and Giovanni Battista Ferrero ${ }^{1}$ \\ 1 Department of Public Health and Pediatric Sciences, University of Torino, 10126 Torino, Italy; \\ valentina.ciuffreda@edu.unito.it (V.P.C.); pina.sauro@edu.unito.it (P.S.); veronica.pagliardini@libero.it (V.P.); \\ pagliardini.severo@virgilio.it (S.P.); diana.carli@unito.it (D.C.); franca.fagioli@unito.it (F.F.); \\ epavanello@cittadellasalute.to.it (E.P.); giovannibattista.ferrero@unito.it (G.B.F.) \\ 2 Neonatal Intensive Care Unit, Department of Obstetrics and Gynecology, S.Anna Hospital, Città della Salute \\ e della Scienza, 10126 Torino, Italy \\ 3 Division of Human Genetics, the Children's Hospital of Philadelphia and Department of Pediatrics, \\ The Perelman School of Medicine, University of Pennsylvania, Philadelphia, PA 19104, USA; \\ kalishj@email.chop.edu \\ 4 Pediatric Onco-Hematology, Stem Cell Transplantation and Cellular Therapy Division, Regina Margherita \\ Children's Hospital, Città della Salute e della Scienza, 10126 Torino, Italy \\ * Correspondence: alessandro.mussa@unito.it; Tel.: +39-011-313-1985; Fax: +39-011-313-4888
}

Received: 11 November 2018; Accepted: 11 January 2019; Published: 14 January 2019

\begin{abstract}
Background: Hepatoblastoma screening in the Beckwith-Wiedemann spectrum (BWSp) is currently based on measuring a specific serum marker alpha-fetoprotein $(\alpha \mathrm{FP})$ every three months until the fourth birthday. Frequent blood draws can be a burden for patients and their families. Methods: We have developed a less invasive alternative testing method based on measuring $\alpha$ FPs from dried blood spots (DBS). The method was validated with 259 simultaneous plasma and DBS $\alpha$ FP measurements in 171 children (132 controls and 39 patients with BWSp). Results: The DBS and plasma measurements overlapped across the wide range of $\alpha \mathrm{FP}$ concentrations independent of patient age $(p<0.0001)$, demonstrating the utility of this method for longitudinal monitoring. Occasional differences between measurements by the two techniques fell within standard laboratory error and would not alter clinical management. Conclusions: This novel method shows consistent overlap with the traditional blood draws, thereby demonstrating its utility for hepatoblastoma screening in this setting and alleviating the burden of frequent blood draws. This also may help increase patient compliance and reduce costs of health care screening. The DBS-based method for the measurement of cancer biomarkers may also be applied to several other chronic diseases with increased risks of $\alpha \mathrm{FP}$-producing liver tumors.
\end{abstract}

Keywords: alpha-fetoprotein; Beckwith-Wiedemann syndrome; dried blood spot; hepatoblastoma; screening

\section{Introduction}

The Beckwith-Wiedemann spectrum (BWSp) consists of the variable association of macroglossia, abdominal wall defects, organomegaly, ear pits/creases, facial nevus simplex, hyperinsulinemic hypoglycemia, lateralized overgrowth, and embryonal tumor predisposition [1-3]. BWSp includes the classical Beckwith-Wiedemann syndrome (BWS, OMIM \#130650), the most common overgrowth and cancer predisposition disorder (1:10,500 live births) [4], and more subtle presentations with an 
11p15.5 molecular anomaly, including Isolated Lateralized Overgrowth (ILO, OMIM \#235000) [5]. The BWSp embryonal tumor predisposition in childhood includes an increased risk of developing hepatoblastoma (HB), which occurs in up to $3.5 \%$ of patients depending on the specific genetic anomaly [6,7]. HB typically occurs before 30 months of age with a peak of incidence at six months $[8,9]$, and BWS represents the most relevant risk factor for $\mathrm{HB}$, with a relative risk of 2280 times greater than in the general population [10]. The molecular subgroups of BWS patients with the highest risk for HB are those with paternal uniparental disomy (UPD) of chromosome 11 or genome-wide UPD [11-13]. Moreover, $\mathrm{HB}$ is the most common tumor diagnosed in BWSp children with the loss of methylation at imprinting control region 2, the molecular subgroup representing approximately $50 \%$ of BWSp patients [14].

HB usually grows rapidly; therefore, survival and prognosis are highly dependent on early diagnosis $[8,9,15]$. More than $95 \%$ of HB secrete the highly sensitive and specific tumor marker alpha-fetoprotein $(\alpha \mathrm{FP})[9,15]$, and measuring $\alpha$ FPs are used in diagnosis, follow-up, and relapse detection. BWSp patients are monitored for HB with $\alpha$ FPs every three months from birth to the fourth birthday [16-19]. Screening provides the opportunity for early detection and at earlier stages of the diagnosis, potentially allowing for less toxic therapies [20,21].

However, $\alpha \mathrm{FP}$ screening in this population has been controversial for a number of reasons $[6,7,14,22-24]$. First, the variability in the physiologic decrease of normal serum $\alpha F P$ levels (from $10^{5} \mathrm{U} / \mathrm{mL}$ magnitude at birth to concentrations steadily $<10 \mathrm{U} / \mathrm{mL}$ by the age of $12-24$ months) leads to challenges in the interpretation of $\alpha \mathrm{FP}$ levels in early infancy [25,26]. Moreover, normal $\alpha \mathrm{FP}$ values in BWS patients in the first year of life tend to be elevated compared with normal pediatric values [27]. Age-corrected reference values should be employed $[25,26]$, and $\alpha \mathrm{FP}$ trends, rather than reliance of the actual value compared to non-BWS norms, is a more accurate screening strategy $[16,23,28]$.

Second, some health care providers consider the incidence of $\mathrm{HB}$ too low to warrant specific screening [2]: While recently the American Association for Cancer Research (AACR) Childhood Cancer Predisposition Workshop adopted a 1\% risk threshold for surveillance and recommended $\alpha \mathrm{FP}$ screening for all cases of BWSp [29], a consensus statement from the European Network of Human Congenital Imprinting Disorders (EUCID) judged a 5\% threshold to be more appropriate for European healthcare systems and did not recommend $\alpha \mathrm{FP}$ screening in these patients [2].

Lastly, the frequent blood draw schedule in early infancy is perceived as invasive, represents a burden for some children and families, and may cause compliance issues [22,23]. However, parents report being comforted and reassured by the screening [30]. For this reason, in a previous report we demonstrated the technical feasibility of $\alpha \mathrm{FP}$ determination using dried capillary blood spots (DBS) [31]. Here we demonstrate the utility of DBS in parallel to the currently accepted practice of venous $\alpha \mathrm{FP}$ sampling in a range of BWSp patients and normal controls. The method described in our previous report was introduced as a clinical practice pilot program in our institution and compared in parallel to the standard laboratory method. In this report, we describe our experience in the longitudinal monitoring of BWSp by the novel method, further supporting its utility in routine clinical practice.

\section{Results}

Measurements on plasma and DBS were closely correlated $\left(r^{2}=0.999, p<0.001\right.$, Figure 1). Raw measurement data are provided in Supplementary Table S1. The $\alpha \mathrm{FP}$ measurements by the two methods showed consistency and largely overlapped across the wide range of physiological concentrations of the tumor marker $(0.3-97,198.0 \mathrm{U} / \mathrm{mL}$ in plasma and from 0.1 to $97,889.0 \mathrm{U} / \mathrm{mL}$ on DBS). 


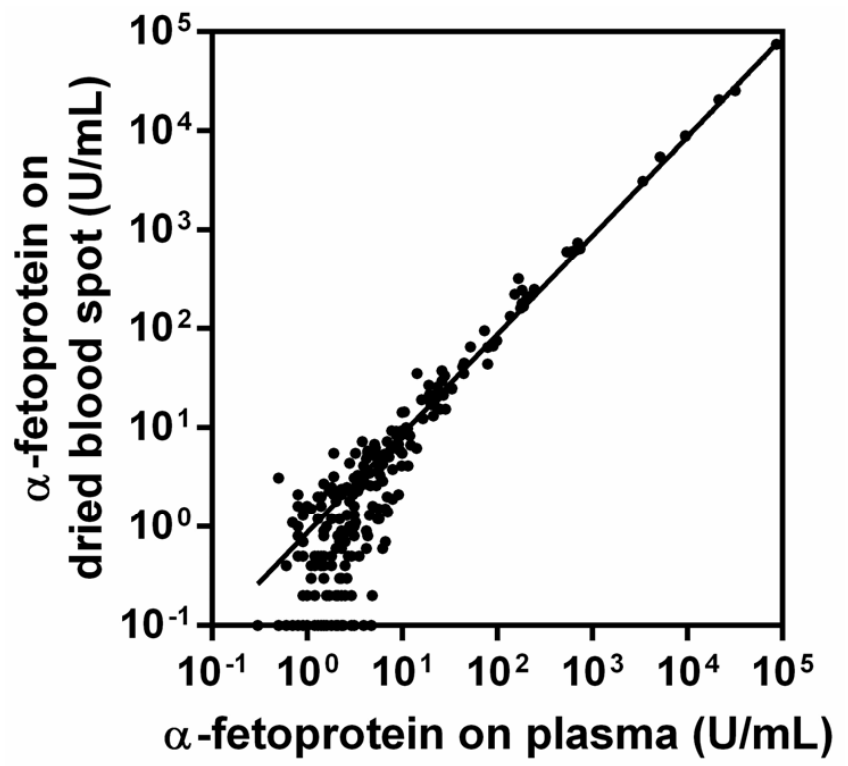

Figure 1. Correlation between $\alpha$-fetoprotein ( $\alpha \mathrm{PF}$ ) measured on plasma and on dried blood spot (DBS) $\left(r^{2}=0.999, p<0.001\right)$.

In 202 cases, both methods measured $\alpha \mathrm{FP} \leq 10 \mathrm{U} / \mathrm{mL}$, whereas in 51 cases, both measurements were $>10 \mathrm{U} / \mathrm{mL}$. In five cases, $\alpha \mathrm{FP}$ was $>10 \mathrm{U} / \mathrm{mL}$ in plasma and $\leq 10 \mathrm{U} / \mathrm{mL}$ on DBS: Patient 3 with $11.4 \mathrm{U} / \mathrm{mL}$ on plasma and $9.8 \mathrm{U} / \mathrm{mL}$ on DBS, Patient 4 with $11.5 \mathrm{U} / \mathrm{mL}$ on plasma and $4.1 \mathrm{U} / \mathrm{mL}$ on DBS, and Patient 5 with $12.0 \mathrm{U} / \mathrm{mL}$ on plasma and $8.3 \mathrm{U} / \mathrm{mL}$ on DBS. In one case, serum $\alpha \mathrm{FP}$ was $\leq 10 \mathrm{U} / \mathrm{mL}(9.9 \mathrm{U} / \mathrm{mL})$ and $>10 \mathrm{U} / \mathrm{mL}$ on DBS $(14.3 \mathrm{U} / \mathrm{mL})$. All the paired measurements show differences between serum and DBS measurements within the coefficient of variation (CV\%). Twenty-six patients and 20 controls had more than one $\alpha \mathrm{FP}$ measurement; 22 patients and 123 controls had 1 paired measurement.

Of the 26 patients with a longitudinal assessment, 12 patients had $\alpha \mathrm{FP}$ measurements $>10 \mathrm{U} / \mathrm{mL}$ : Their $\alpha$ FP trend over time is displayed in Figure 2 to highlight the concordance between the two methods. The remaining 14 patients were assessed longitudinally, and all had concordant DBS-plasma $\alpha \mathrm{FP}<10 \mathrm{U} / \mathrm{mL}$ over time (not shown as values under $10 \mathrm{U} / \mathrm{mL}$ are considered within normal range). Of the 22 patients tested with a single paired measurement, 19 had concordant DBS-plasma $\alpha \mathrm{FP}$ values $<10 \mathrm{U} / \mathrm{mL}$ and three had values $>10 \mathrm{U} / \mathrm{mL}$. In the latter group, Patient 29 showed $225.0 \mathrm{U} / \mathrm{mL}$ in plasma and 212.0 U/mL on DBS, Patient 30 had $609.0 \mathrm{U} / \mathrm{mL}$ in plasma and $610.5 \mathrm{U} / \mathrm{mL}$ on DBS, and Patient 37 had $187.1 \mathrm{U} / \mathrm{mL}$ in plasma and $168.0 \mathrm{U} / \mathrm{mL}$ on DBS. The patient diagnosed with non-syndromic $\mathrm{HB}$, a female aged 31 months, had an $\alpha \mathrm{FP}$ of $583.5 \mathrm{U} / \mathrm{mL}$ measured by the traditional method and $601.0 \mathrm{U} / \mathrm{mL}$ on DBS. 

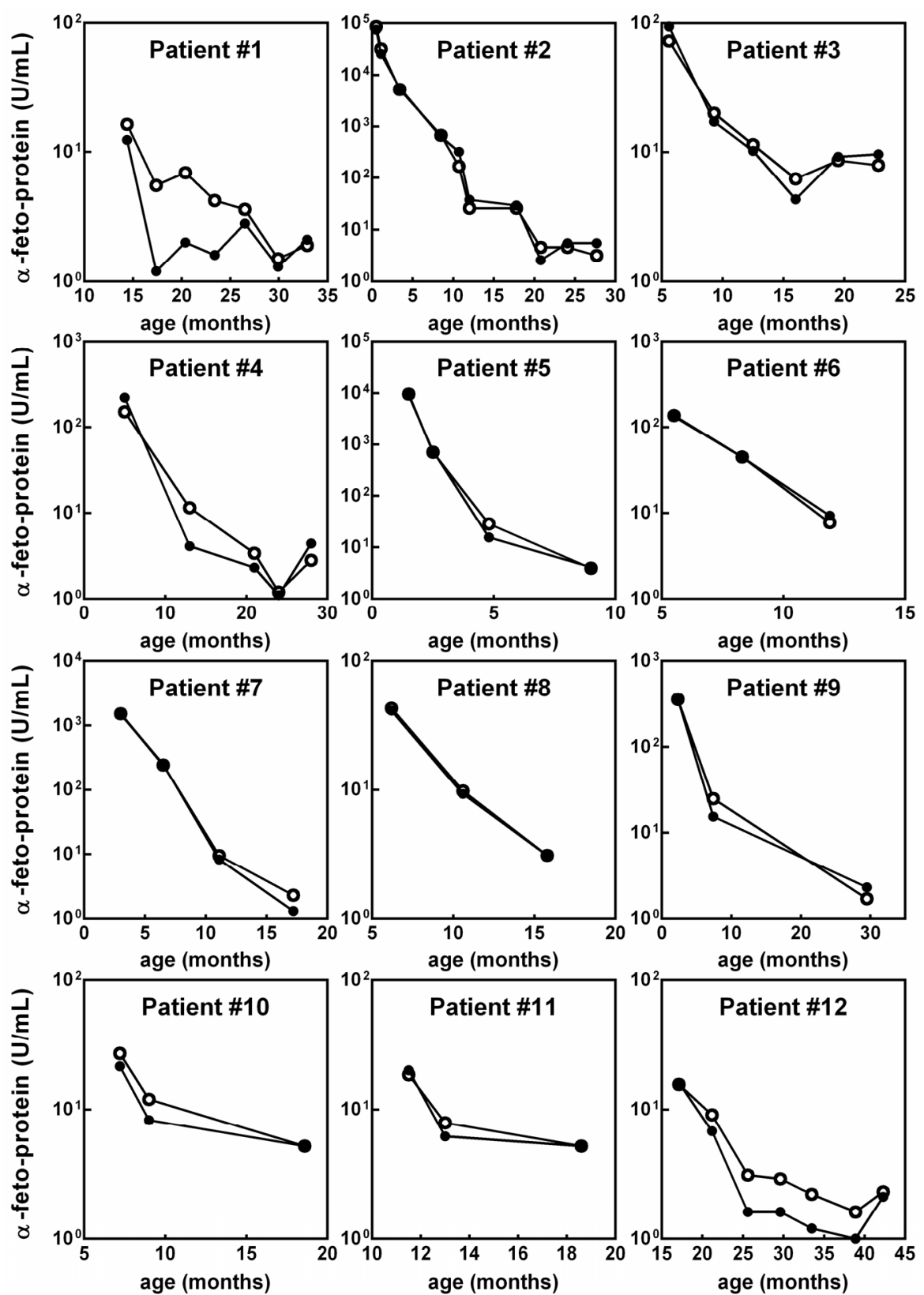

Figure 2. The longitudinal evaluation of alpha-fetoprotein $(\alpha \mathrm{FP})$ plasmatic concentration on dried blood spots (DBS, closed circles) overlapped that on standard laboratory method (open circles) in the 12 patients affected by cancer-predisposition syndromes with $\alpha \mathrm{FP}$ concentrations $>10 \mathrm{U} / \mathrm{mL}$.

\section{Discussion}

In this work, we demonstrate the feasibility of HB screening in overgrowth-cancer predisposition syndromes using DBS for $\alpha \mathrm{FP}$ measurements. DBS, a novel method to measure $\alpha \mathrm{FP}$ concentration showed consistent overlap with the traditional venous sampling method, showing reliability in the clinical setting of a tumor screening program. In the first two years of life-those in which the likelihood of developing HB is commonly higher in such conditions [9] $-\alpha \mathrm{FP}$ concentrations decrease rapidly from a $10^{6}-10^{5}$ to a $<10 \mathrm{U} / \mathrm{mL}$ magnitude, with almost unpredictable and variable timing, making interpretations challenging. Although age-specific and gestational age-corrected normal $\alpha \mathrm{FP}$ concentrations values are available [32], HB screening relies mostly on the longitudinal observation of repeated measurements of $\alpha \mathrm{FP}$ concentrations rather than on the detection of a single measurement $[28,29]$. The DBS measurements overlapped the serum measurements across a wide 
range of physiologic concentrations and ages, demonstrating the utility of our methodology for longitudinal monitoring in both newborns and toddlers. Moreover, the range of measurements includes a $10^{5}$ magnitude often observed in prematurity [32], which is common in the BWSp [33].

After the physiological decrease of $\alpha \mathrm{FP}$ serum concentrations, a cutoff of $>10 \mathrm{U} / \mathrm{mL}$ is commonly used to define normal values after 2 years of age. The measurement provided by the traditional and novel methods consistently matched across this diagnostic threshold, allowing, therefore, the determination of normal screens compared to abnormal screening tests. The few cases with discordant plasma and DBS measurements showed very tight fluctuations across the $10 \mathrm{U} / \mathrm{mL}$ threshold within the acceptable error range for these tests, and clinical management was not altered by these fluctuations.

Additionally, our patient who was ultimately diagnosed with a non-syndromic HB, showed that $\alpha \mathrm{FP}$ levels determined by the two methods were highly consistent in the setting of a tumor diagnosis as well. Hence, we propose using DBS for HB screening and for patients presenting abnormal results suggesting a tumor diagnosis; further investigation with both conventional venous $\alpha \mathrm{FP}$ testing and imaging studies should be used to confirm or exclude a HB diagnosis. Finally, the DBS technique alleviates the burden of frequent blood draws and is simple, efficient and low-cost, thus making the routine measurement of $\alpha \mathrm{FP}$ more practical. These aspects are crucial to improve patients' compliance with tumor surveillance recommendations for cancer predisposition syndromes. It has recently been shown that accurate knowledge about cancer risk and screening in the BWSp context decreases parents' worries about tumor development [30] and that the DBS method may also decrease children's anxiety related to HB screening. The DBS method is cheaper in terms of storage, transport, and handling even compared to other minimally invasive methods (i.e., finger poke in microtainer tube) that require that the samples remain in a liquid state [34]. DBS home sampling is associated with a reduction in costs both from a healthcare and from a societal perspective with patient costs abated nearly to zero and with a relevant decrease in costs related to the loss of productivity [35].

Other potential applications of the DBS method may come from this work and include screening of other conditions with increased risk of hepatocarcinoma or other $\alpha \mathrm{FP}$-secreting tumors as well as follow-up of patients treated for liver tumors. As screening for hepatocarcinoma in cirrhotic patients by repeated serum $\alpha \mathrm{FP}$ measurement is feasible [36] and results in increased survival rates [28,37], monitoring in cirrhotic patients by DBS may represent a specific example of future applications.

\section{Materials and Methods}

\subsection{Patients}

Overall, 259 simultaneous plasma and DBS $\alpha$ FP measurements were performed in 171 children (mean age $38.7 \pm 59.2$ months, range 0-7.3 years, 88 males, 83 females). Of these, 116 paired measurements have been performed in 48 patients with syndromes with increased risk of $\mathrm{HB}$ for tumor screening (range 0-60 months): 39 were affected by BWSp/ILO (23 molecularly confirmed, 16 diagnosed clinically with negative molecular tests), 3 had macrocephaly-capillary malformation syndrome, 5 had undiagnosed likely syndromic overgrowth disorders, and one girl had an isolated HB. Of these, 31 measurements from 31 patients were previously reported in our preliminary report [31]. The remaining 143 paired measurements were performed in 123 children as controls: 27 were healthy children and 96 underwent blood tests for suspected conditions with no effect on plasmatic $\alpha \mathrm{FP}$ concentration (20 suspected or well compensated thyroid disorders, 35 with recently healed infections, 12 serum lipids screening, 13 affected by phenylketonuria, 10 suspected iron deficient anemia, and 6 suspected precocious puberty). Besides studying the potential utility of the DBS method for longitudinal monitoring, we applied the novel method to patients admitted for suspected abdominal tumors. Twenty-three paired measurements were therefore performed in patients referred to our Division of Pediatric Oncology for suspected neoplasms and who had $\alpha$ FP measurement with the aim to identify potential $\alpha$ FP producing tumors. Among the last cases, the suspected diagnosis of 
neoplasm was excluded after the tests (including $\alpha \mathrm{FP}$ and appropriate imaging) except for one female who was ultimately diagnosed with non-syndromic HB.

\subsection{Study and Screening Protocol}

Informed consent was obtained from parents using a study protocol approved by the Institutional Review Board of our University Hospital (IRB number CS/156/2014 Città della Salute e della Scienza di Torino, University of Torino, Italy). Our protocol for HB screening in overgrowth-cancer predisposition syndromes is consistent with that proposed by the American Association for Cancer Research [29] and is based on 3 months' abdominal ultrasound and simultaneous $\alpha$ FP measurements by standard laboratory methodology from birth up to the fourth birthday [17]. A negative $\alpha \mathrm{FP}$ test is defined as an $\alpha$ FP measurement less than $10 \mathrm{U} / \mathrm{mL}(1 \mathrm{U} / \mathrm{mL}=1.21 \mathrm{ng} / \mathrm{mL})$ or declining with respect to previous measurement. The individual value is interpreted in the context of the $\alpha \mathrm{FP}$ trend over time, with an expectation of declining values through infancy. In case of concentrations $>10 \mathrm{U} / \mathrm{mL}$, the results need to be interpreted on the basis of normal BWS values (which tend to be elevated over the first years of life compared with normal pediatric values), with age-specific and gestational age-corrected reference values provided in the literature [25] and with previous measurements performed in the same patient, if available. If the concentrations are less than the previous ones, then we consider the test negative and register the absolute value in order to perform subsequent comparisons. If we detect an $\alpha \mathrm{FP}$ greater than the previous one, the test is referred to as positive, recent imaging is re-evaluated, and the patient is recalled for subsequent $\alpha \mathrm{FP}$ remeasurements after a 6-week interval for rises greater than 50-100 U/mL [29]. In cases of significantly larger increases (greater than $1000 \mathrm{U} / \mathrm{mL}$ ) or with further increase at the 6 weeks $\alpha \mathrm{FP}$ remeasurement, second-step medical investigations are proposed (targeted liver US or MRI) [29].

\subsection{Laboratory Assays}

Paired $\alpha$ FP measurements were simultaneously performed on blood, obtained by venipuncture, and DBS, collected by heel-stick or by spotting single blood drops from a syringe directly onto standard filter paper employed for newborn screening. The DBS specimens were dried at room temperature, routinely stored in plastic bags at $4{ }^{\circ} \mathrm{C}$ and analyzed employing a $3.2 \mathrm{~mm}$-diameter spotted filter paper punch containing approximately $3.4 \mu \mathrm{L}$ of adsorbed blood. The serum $\alpha \mathrm{FP}$ measurement kit (AutoDELFIA hAFP, Perkin Elmer, Waltham, Massachusetts) adapted to the DBS technique has been employed, as previously described [31]. The intra-assay CV\% was evaluated for quality controls: For low concentrations $(\sim 10 \mathrm{U} / \mathrm{mL})$, the $\mathrm{CV} \%$ s were $3.02 \%$ for the plasma assay and $4.11 \%$ for the DBS one; for high concentrations quality controls ( $70 \mathrm{U} / \mathrm{mL})$, the CV\%s were $3.05 \%$ and $3.22 \%$, respectively.

\subsection{Statistics}

Data were analysed by GraphPad Prism 6.0 (GraphPad Software, Inc. La Jolla, CA, USA). Data distribution was assessed by the Shapiro-Wilk test and correlations tested by Pearson or Sperman methods, accordingly.

\section{Conclusions}

In conclusion, in this study we demonstrate that screening children with overgrowth disorders with HB predispositions can be performed by a novel simple method, which measures $\alpha$ PF on DBS. This novel technique may lead to increased adherence and reduced anxiety and cost.

Supplementary Materials: The following are available online at http:/ /www.mdpi.com/2072-6694/11/1/86/s1, Table S1: Raw data of the paired measurements of alpha-fetoprotein $(\alpha \mathrm{FP})$ with the traditional and dried blood spot (DBS) methods. 
Author Contributions: A.M. designed the study, analyzed and interpreted the results, and drafted the manuscript; S.P. ideated the method, wrote the methods section, and revised the manuscript; E.P., V.P.C., and P.S. performed the blood assays, managed the samples, contributed to the drafting, and revised the paper; V.P. and D.C. collected the samples, contributed to data collection, wrote parts of the results, and revised the manuscript; J.M.K. and F.F. provided some of the samples, contributed to data analysis and interpretation, and revised critically the manuscript; and G.B.F. contributed to the study design and to the drafting of the manuscript, provided funding for sample analysis, and revised critically the manuscript.

Funding: The study was supported by the grant sponsor by MIUR (Italian Ministry for Education, University and Research) PRIN 2009-MBHZPR to G.B.F. and by National Institutes of Health (K08 CA193915), Alex's Lemonade Stand Foundation, and St. Baldrick's Foundation to J.M.K.

Conflicts of Interest: The authors declare no conflict of interest.

\section{References}

1. Brioude, F.; Kalish, J.M.; Mussa, A.; Foster, A.C.; Bliek, J.; Ferrero, G.B.; Boonen, S.E.; Cole, T.; Baker, R.; Bertoletti, M.; et al. Expert consensus document: Clinical and molecular diagnosis, screening and management of Beckwith-Wiedemann syndrome: An international consensus statement. Nat. Rev. Endocrinol. 2018, 14, 229-249. [CrossRef] [PubMed]

2. Mussa, A.; Russo, S.; Larizza, L.; Riccio, A.; Ferrero, G.B. (Epi)genotype-phenotype correlations in Beckwith-Wiedemann syndrome: A paradigm for genomic medicine. Clin. Genet. 2016, 89, 403-415. [CrossRef]

3. Mussa, A.; Russo, S.; De Crescenzo, A.; Freschi, A.; Calzari, L.; Maitz, S.; Macchiaiolo, M.; Molinatto, C.; Baldassarre, G.; Mariani, M.; et al. (Epi)genotype-phenotype correlations in Beckwith-Wiedemann syndrome. Eur. J. Hum. Genet. 2016, 24, 183-190. [CrossRef] [PubMed]

4. Mussa, A.; Russo, S.; De Crescenzo, A.; Chiesa, N.; Molinatto, C.; Selicorni, A.; Richiardi, L.; Larizza, L.; Silengo, M.C.; Riccio, A.; et al. Prevalence of Beckwith-Wiedemann syndrome in North West of Italy. Am. J. Med. Genet. A 2013, 161A, 2481-2486. [CrossRef] [PubMed]

5. Kalish, J.M.; Biesecker, L.G.; Brioude, F.; Deardorff, M.A.; Di Cesare-Merlone, A.; Druley, T.; Ferrero, G.B.; Lapunzina, P.; Larizza, L.; Maas, S.; et al. Nomenclature and definition in asymmetric regional body overgrowth. Am. J. Med. Genet. A 2017. [CrossRef] [PubMed]

6. Maas, S.M.; Vansenne, F.; Kadouch, D.; Ibrahim, A.; Bliek, J.; Hopman, S.; Mannens, M.M.; Merks, J.H.; Maher, E.R.; Hennekam, R.C. Phenotype, cancer risk, and surveillance in Beckwith-Wiedemann syndrome depending on molecular genetic subgroups. Am. J. Med. Genet. A 2016, 170, 2248-2260. [CrossRef] [PubMed]

7. Mussa, A.; Molinatto, C.; Baldassarre, G.; Riberi, E.; Russo, S.; Larizza, L.; Riccio, A.; Ferrero, G.B. Cancer Risk in Beckwith-Wiedemann Syndrome: A Systematic Review and Meta-Analysis Outlining a Novel (Epi)Genotype Specific Histotype Targeted Screening Protocol. J. Pediatr. 2016, 176, 142-149. [CrossRef] [PubMed]

8. Spector, L.G.; Birch, J. The epidemiology of hepatoblastoma. Pediatr. Blood Cancer 2012, 59, $776-779$. [CrossRef]

9. Mussa, A.; Duffy, K.A.; Carli, D.; Ferrero, G.B.; Kalish, J.M. Defining an optimal time window to screen for hepatoblastoma in children with Beckwith-Wiedemann syndrome. Pediatr. Blood Cancer 2019, 66, e27492. [CrossRef]

10. DeBaun, M.R.; Tucker, M.A. Risk of cancer during the first four years of life in children from The Beckwith-Wiedemann Syndrome Registry. J. Pediatr. 1998, 132, 398-400. [CrossRef]

11. Mussa, A.; Ferrero, G.B.; Ceoloni, B.; Basso, E.; Chiesa, N.; De Crescenzo, A.; Pepe, E.; Silengo, M.; de Sanctis, L. Neonatal hepatoblastoma in a newborn with severe phenotype of Beckwith-Wiedemann syndrome. Eur. J. Pediatr. 2011, 170, 1407-1411. [CrossRef]

12. Smith, A.C.; Shuman, C.; Chitayat, D.; Steele, L.; Ray, P.N.; Bourgeois, J.; Weksberg, R. Severe presentation of Beckwith-Wiedemann syndrome associated with high levels of constitutional paternal uniparental disomy for chromosome 11p15. Am. J. Med. Genet. A 2007, 143A, 3010-3015. [CrossRef] [PubMed]

13. Kalish, J.M.; Conlin, L.K.; Bhatti, T.R.; Dubbs, H.A.; Harris, M.C.; Izumi, K.; Mostoufi-Moab, S.; Mulchandani, S.; Saitta, S.; States, L.J.; et al. Clinical features of three girls with mosaic genome-wide paternal uniparental isodisomy. Am. J. Med. Genet. A 2013, 161A, 1929-1939. [CrossRef] 
14. Duffy, K.A.; Deardorff, M.A.; Kalish, J.M. The utility of alpha-fetoprotein screening in Beckwith-Wiedemann syndrome. Am. J. Med. Genet. A 2017, 173, 581-584. [CrossRef] [PubMed]

15. Czauderna, P.; Lopez-Terrada, D.; Hiyama, E.; Häberle, B.; Malogolowkin, M.H.; Meyers, R.L. Hepatoblastoma state of the art: Pathology, genetics, risk stratification, and chemotherapy. Curr. Opin. Pediatr. 2014, 26, 19-28. [CrossRef] [PubMed]

16. Zarate, Y.A.; Mena, R.; Martin, L.J.; Steele, P.; Tinkle, B.T.; Hopkin, R.J. Experience with hemihyperplasia and Beckwith-Wiedemann syndrome surveillance protocol. Am. J. Med. Genet. A 2009, 149A, 1691-1697. [CrossRef] [PubMed]

17. Mussa, A.; Di Candia, S.; Russo, S.; Catania, S.; De Pellegrin, M.; Di Luzio, L.; Ferrari, M.; Tortora, C.; Meazzini, M.C.; Brusati, R.; et al. Recommendations of the Scientific Committee of the Italian Beckwith-Wiedemann Syndrome Association on the diagnosis, management and follow-up of the syndrome. Eur. J. Med. Genet. 2016, 59, 52-64. [CrossRef]

18. Tan, T.Y.; Amor, D.J. Tumour surveillance in Beckwith-Wiedemann syndrome and hemihyperplasia: A critical review of the evidence and suggested guidelines for local practice. J. Paediatr. Child. Health 2006, 42, 486-490. [CrossRef]

19. Bliek, J.; Maas, S.; Alders, M.; Merks, J.H.; Mannens, M. Epigenotype, phenotype, and tumors in patients with isolated hemihyperplasia. J. Pediatr. 2008, 153, 95-100. [CrossRef] [PubMed]

20. Trobaugh-Lotrario, A.D.; Venkatramani, R.; Feusner, J.H. Hepatoblastoma in children with Beckwith-Wiedemann syndrome: Does it warrant different treatment? J. Pediatr. Hematol. Oncol. 2014, 36, 369-373. [CrossRef] [PubMed]

21. Clericuzio, C.L.; Chen, E.; McNeil, D.E.; O'Connor, T.; Zackai, E.H.; Medne, L.; Tomlinson, G.; DeBaun, M. Serum alpha-fetoprotein screening for hepatoblastoma in children with Beckwith-Wiedemann syndrome or isolated hemihyperplasia. J. Pediatr. 2003, 143, 270-272. [CrossRef]

22. Mussa, A.; Ferrero, G.B. Screening Hepatoblastoma in Beckwith-Wiedemann Syndrome: A Complex Issue. J. Pediatr. Hematol. Oncol. 2015, 37, 627. [CrossRef] [PubMed]

23. Mussa, A.; Ferrero, G.B. Serum alpha-fetoprotein screening for hepatoblastoma in Beckwith-Wiedemann syndrome. Am. J. Med. Genet. A 2017, 173, 585-587. [CrossRef] [PubMed]

24. Kalish, J.M.; Deardorff, M.A. Tumor screening in Beckwith-Wiedemann syndrome-To screen or not to screen? Am. J. Med. Genet. A 2016, 170, 2261-2264. [CrossRef] [PubMed]

25. Blohm, M.E.; Vesterling-Hörner, D.; Calaminus, G.; Göbel, U. Alpha 1-fetoprotein (AFP) reference values in infants up to 2 years of age. Pediatr. Hematol. Oncol. 1998, 15, 135-142. [CrossRef]

26. Schneider, D.T.; Calaminus, G.; Göbel, U. Diagnostic value of alpha 1-fetoprotein and beta-human chorionic gonadotropin in infancy and childhood. Pediatr. Hematol. Oncol. 2001, 18, 11-26. [CrossRef] [PubMed]

27. Everman, D.B.; Shuman, C.; Dzolganovski, B.; O'riordan, M.A.; Weksberg, R.; Robin, N.H. Serum alpha-fetoprotein levels in Beckwith-Wiedemann syndrome. J. Pediatr. 2000, 137, 123-127. [CrossRef] [PubMed]

28. Arrieta, O.; Cacho, B.; Morales-Espinosa, D.; Ruelas-Villavicencio, A.; Flores-Estrada, D.; Hernández-Pedro, N. The progressive elevation of alpha fetoprotein for the diagnosis of hepatocellular carcinoma in patients with liver cirrhosis. BMC Cancer 2007, 7, 28. [CrossRef] [PubMed]

29. Kalish, J.M.; Doros, L.; Helman, L.J.; Hennekam, R.C.; Kuiper, R.P.; Maas, S.M.; Maher, E.R.; Nichols, K.E.; Plon, S.E.; Porter, C.C.; et al. Surveillance Recommendations for Children with Overgrowth Syndromes and Predisposition to Wilms Tumors and Hepatoblastoma. Clin. Cancer Res. 2017, 23, e115-e122. [CrossRef] [PubMed]

30. Duffy, K.A.; Grand, K.L.; Zelley, K.; Kalish, J.M. Tumor Screening in Beckwith-Wiedemann Syndrome: Parental Perspectives. J. Genet. Couns. 2018, 27, 844-853. [CrossRef] [PubMed]

31. Mussa, A.; Pagliardini, S.; Pagliardini, V.; Molinatto, C.; Baldassarre, G.; Corrias, A.; Silengo, M.C.; Ferrero, G.B. $\alpha$-Fetoprotein assay on dried blood spot for hepatoblastoma screening in children with overgrowth-cancer predisposition syndromes. Pediatr. Res. 2014, 76, 544-548. [CrossRef] [PubMed]

32. Blair, J.I.; Carachi, R.; Gupta, R.; Sim, F.G.; McAllister, E.J.; Weston, R. Plasma alpha fetoprotein reference ranges in infancy: Effect of prematurity. Arch. Dis. Child. 1987, 62, 362-369. [CrossRef] [PubMed]

33. Mussa, A.; Russo, S.; de Crescenzo, A.; Freschi, A.; Calzari, L.; Maitz, S.; Macchiaiolo, M.; Molinatto, C.; Baldassarre, G.; Mariani, M.; et al. Fetal growth patterns in Beckwith-Wiedemann syndrome. Clin. Genet. 2016, 90, 21-27. [CrossRef] 
34. McDade, T.W. Development and validation of assay protocols for use with dried blood spot samples. Am. J. Hum. Biol. 2014, 26, 1-9. [CrossRef] [PubMed]

35. Martial, L.C.; Aarnoutse, R.E.; Schreuder, M.F.; Henriet, S.S.; Brüggemann, R.J.; Joore, M.A. Cost Evaluation of Dried Blood Spot Home Sampling as Compared to Conventional Sampling for Therapeutic Drug Monitoring in Children. PLoS ONE 2016, 11, e0167433. [CrossRef] [PubMed]

36. Choi, J.; Kim, G.A.; Han, S.; Lee, W.; Chun, S.; Lim, Y.S. Longitudinal Assessment of Three Serum Biomarkers to Detect Very Early Stage Hepatocellular Carcinoma. Hepatology 2018. [CrossRef] [PubMed]

37. El-Serag, H.B.; Kramer, J.R.; Chen, G.J.; Duan, Z.; Richardson, P.A.; Davila, J.A. Effectiveness of AFP and ultrasound tests on hepatocellular carcinoma mortality in HCV-infected patients in the USA. Gut 2011, 60, 992-997. [CrossRef] [PubMed]

(C) 2019 by the authors. Licensee MDPI, Basel, Switzerland. This article is an open access article distributed under the terms and conditions of the Creative Commons Attribution (CC BY) license (http:/ / creativecommons.org/licenses/by/4.0/). 